\title{
Research on the Heating of Deicing Fluid in a New Reshaped Coiled Tube
}

\author{
Mengli Wu, Chiyu Wang, Yunpeng Li, and Qi Nie \\ College of Aeronautical Engineering, Civil Aviation University of China, Tianjin 300300, China \\ Correspondence should be addressed to Mengli Wu; wuml2004@qq.com
}

Received 19 October 2017; Accepted 7 December 2017; Published 31 December 2017

Academic Editor: Sergey A. Suslov

Copyright (c) 2017 Mengli Wu et al. This is an open access article distributed under the Creative Commons Attribution License, which permits unrestricted use, distribution, and reproduction in any medium, provided the original work is properly cited.

\begin{abstract}
Aircraft ground deicing operation is significant to ensure civil flight safety in winter. Helically coiled tube is the important heat exchanger in Chinese deicing fluid heating system. In order to improve the deicing efficiency, the research focuses on heat transfer enhancement of deicing fluid in the tube. Based on the field synergy principle, a new reshaped tube (TCHC) is designed by ring-rib convex on the inner wall. Deicing fluid is high viscosity ethylene-glycol-based mixture. Because of the power function relation between high viscosity and temperature, viscosity has a negative influence on heat transfer. The number of ring-ribs and inlet velocity are two key parameters to the heat transfer performance. For both water and ethylene glycol, the outlet temperature rises when the number of ring-ribs increases to a certain limit. However, the increasing of velocity reduces heating time, which results in lower outlet temperature. The heating experiment of the original tube is conducted. The error between experiment and simulation is less than $5 \%$. The outlet temperature of TCHC increases by $3.76 \%$. As a result, TCHC efficiently promotes the coordination of velocity and temperature fields by changing the velocity field. TCHC has enhanced heat transfer of high viscosity deicing fluid.
\end{abstract}

\section{Introduction}

In the snow and ice weather situation, ground deicing is significant to the safety of the civil flight. In recent years, aircraft ground centralized deicing has been developed in the world. The main structure of ground deicing fluid heating system [1] made by China is shown in Figure 1. Helically coiled tube is the important heat exchanger in the system. High viscosity deicing fluid needs to be quickly heated in the tube. In order to improve the deicing efficiency and ensure the flight punctuality, it is important to study the heat transfer enhancement of deicing fluid.

The deicing fluid is complex ethylene-glycol-based mixture. Its viscosity changes nonlinearly with the increasing of temperature, which obviously affects heat transfer. Most researches on deicing fluid are mainly about material properties $[2,3]$. However, few papers about heat transfer of have been openly reported. The structure deformation is a commonly used and effective method to heat transfer enhancement. Aiming at rapidly heating deicing fluid, our group has proposed a new tube based on field synergy principle, which is called transverse corrugation helically coiled tube (TCHC).
Heat transfer enhancement has always been a hotspot. Field synergy principle is a novel theory in this aspect, which was recently proposed by GUO, a Chinese scholar. The principle indicates that the intensity of heat transfer is related to not only the velocity and the fluid properties, but also the synergy degree between the velocity and temperature fields [4-6]. The heat transfer enhancement can be achieved by improving the coordination between velocity and temperature fields. Based on this principle, many researches have been carried on the heat transfer enhancement of straight tubes by changing the flow velocity field [7-10]. The principle has been widely tested and applied by many investigators.

Many scholars have studied the flow properties inside the helically coiled tube. Dean $[11,12]$ gave out the NavierStokes function of flow in the helically coiled tube. Resulting from the difference of pressure of flow inside, the secondary flow was proved to improve the heat transfer efficiency. Since late 20th century, the Computational Fluid Dynamics (CFD) technique has developed quickly $[13,14]$. Palanichamy and Nagaraj [15] presented a numerical simulation of laminar heat transfer in circular tube flows with internal longitudinal fins using finite difference numerical code. Tian et al. [16] 


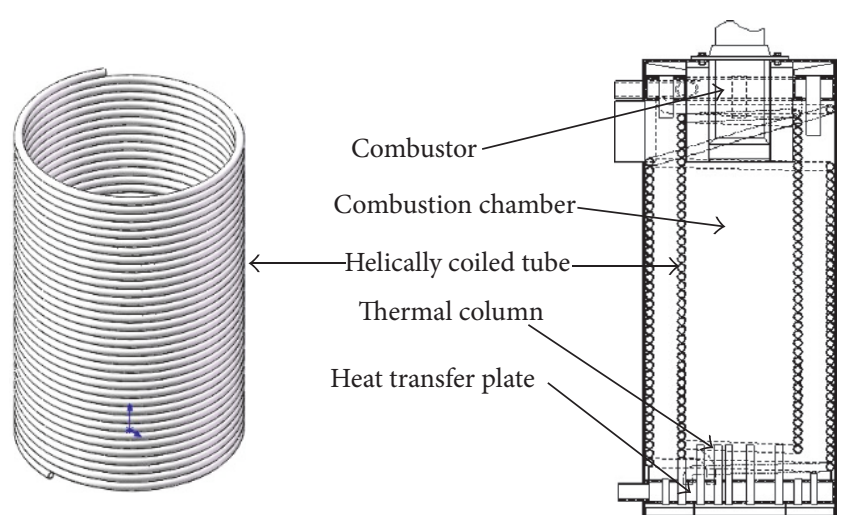

FIGURE 1: The structure of aircraft ground deicing fluid heating system.

investigated numerically the effects of six geometric parameters and pin-fin arrangement in the flow direction on the thermohydraulic performance

Numerical methods have been employed to analyze the influence of geometrical configurations and flow parameters in heat transfer of helically coiled tube. Lin and Ebadian [17] and Di Piazza and Ciofalo [18] studied the effect of the pitch and the curvature of the tube on convective heat transfer. The $k-\varepsilon$ model is a two-equation turbulent model proposed by Jones and Launder in 1972 [19]. The $k-\varepsilon$ model is the most widely used complete turbulence model. It is incorporated in most CFD codes [20]. The realizable $k-\varepsilon$ model, proposed by Shih et al., has been validated in simulation of a variety of flows including rotating homogeneous shear flows, channel flows, and boundary flows [21, 22]. Hüttl and Friedrich [23] indicated that curvature ratio had a strong impact on the secondary flow. Zachár [24] studied the natural convection induced heat transfer over the outer surface of the tube. Different geometrical configurations and flow parameters were considered. A fluid-to-fluid boundary condition was set to realize the actual flow configurations. The results of the inner side heat transfer rate were tested by existing empirical formulas and experimental results.

Aiming at the heat transfer enhancement of deicing fluid, TCHC is designed based on field synergy principle. As ideal fluid, water is studied for contrast on the influence of viscosity. Due to complex properties, the deicing fluid is simplified by its main component, ethylene glycol. The number of ringribs and inlet velocity are two key parameters to the heat transfer performance. These parameters' variation of ethylene glycol and water is simulated in TCHC and the original tube. The outlet temperature is chosen as the evaluation index. The heating experiment is conducted to verify the simulation.

\section{Numerical Method}

For heat transfer enhancement of deicing fluid, original helically coiled tube has been reshaped to TCHC based on field synergy principle.

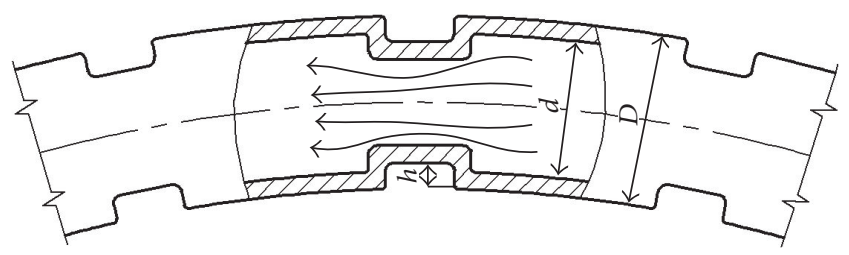

FIgURE 2: Structure of ring-rib inside TCHC.

TABle 1: Parameters of the original tube and TCHC.

\begin{tabular}{lcccc}
\hline Tube type & NO. 1 & NO. 2 & NO. 3 & NO. 4 \\
\hline $\begin{array}{l}\text { Number of } \\
\text { ring-ribs/per round }\end{array}$ & 0 & 4 & 8 & 16 \\
\hline $\begin{array}{l}\text { Inner diameter/d } \\
(\mathrm{mm})\end{array}$ & 14 & 14 & 14 & 14 \\
\hline $\begin{array}{l}\text { Outer diameter/D } \\
(\mathrm{mm})\end{array}$ & 20 & 20 & 20 & 20 \\
\hline $\begin{array}{l}\text { Ring-rib height/h } \\
(\mathrm{mm})\end{array}$ & 0 & 2 & 2 & 2 \\
\hline
\end{tabular}

TABLE 2: Grid quantity.

\begin{tabular}{lcccc}
\hline Tube type & NO. 1 & NO. 2 & NO. 3 & NO. 4 \\
\hline Grid quantity $\left(10^{4}\right)$ & 76 & 93 & 112 & 134 \\
\hline
\end{tabular}

2.1. Geometrical Configurations. The ring-rib is equal-pitch convex on the inner wall of TCHC. The structure of ringrib inside TCHC has been presented in Figure 2. The flow velocity changes when passing by the ring-rib. Thus, an additional disturbance is generated to enhance heat transfer.

In order to study how ring-rib affects heat transfer, three types of TCHC are obtained by changing the number of ringribs. The original tube is set as NO. 1 tube for contrast. TCHC with different numbers of ring-ribs are set as No. 2 to No. 4 tube. The models of the original tube and TCHC are shown in Figure 3. The detailed parameters of four types of tubes are shown in Table 1.

2.2. Grid Generation. Structural grid technology is used to mesh three different grid models in hexahedral structure. The grid generations of TCHC and the original tube are displayed in Figure 4 . The thickness of wall is simplified, so the section by which the fluid passes is meshed. To show the mesh of tube clearly, solid simple display is a method by making the hollow tube look like solid tube.

The grid quantities of TCHC with different numbers of ring-ribs are given in Table 2 . The grid distribution is uniform which satisfies the requirement of numerical simulation. To make the calculation more accurate, the mesh of ring-rib is more compact than the other parts.

For the original tube and TCHC, the skew values of grid are tested. More than $97 \%$ of grid is within 0.4 . The grid meshing meets the requirement of the calculation. 


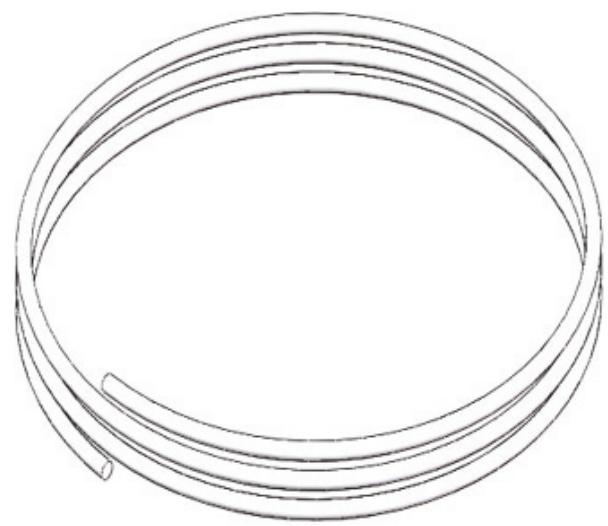

(a)

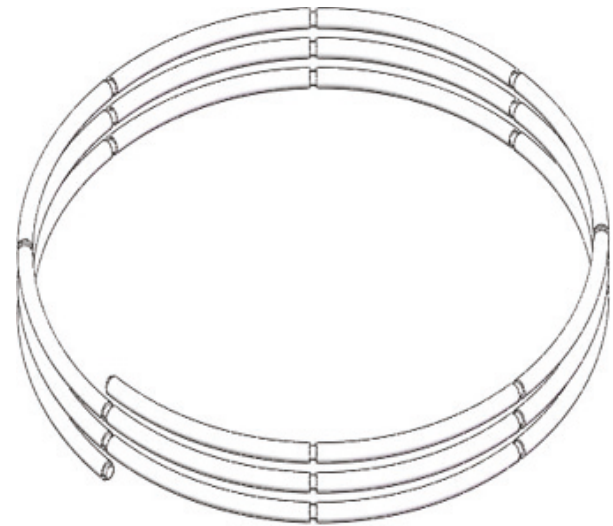

(c)

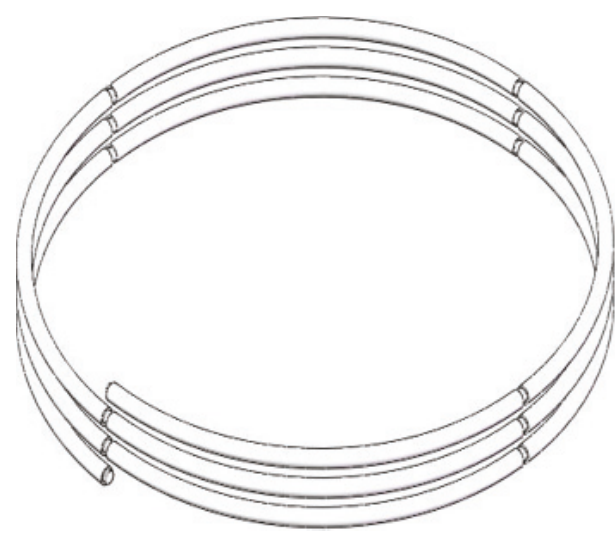

(b)

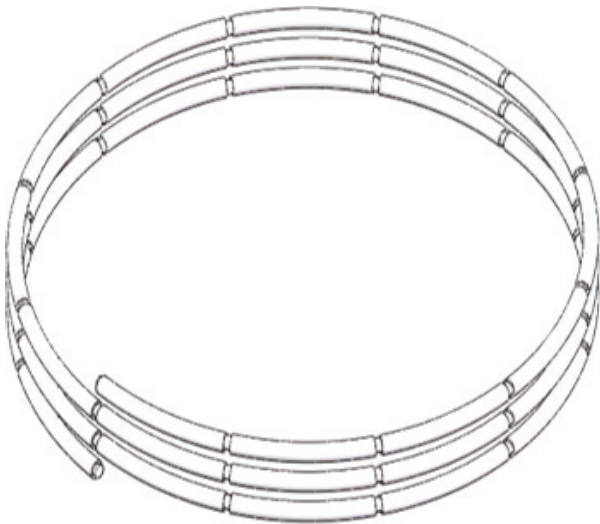

(d)

FIgURE 3: Geometric models of the original tube and TCHC. (a) NO. 1 tube, (b) NO. 2 tube, (c) NO. 3 tube, and (d) NO. 4 tube.

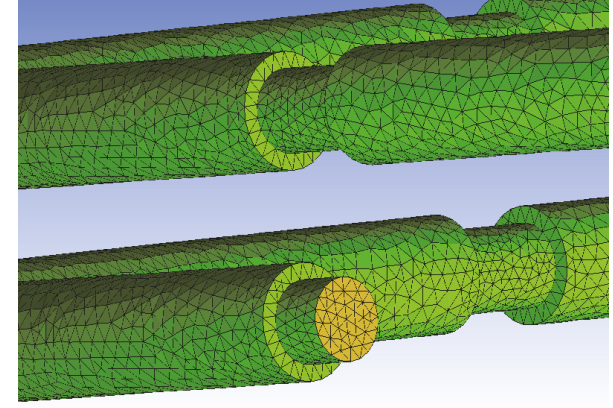

(a)

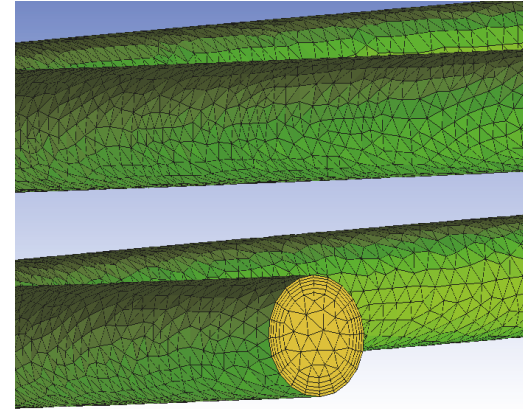

(b)

FIGURE 4: The gird generation (solid simple display). (a) TCHC and (b) the original tube.

\section{Governing Equations and Boundary Conditions}

3.1. Conservation Equations. The basic equations of fluid mechanics include continuity equation, momentum equation, and energy equation, presented as (1) and (3) according to White [25]. On the basis of a large number of experiments, the main idea of CFD is to construct different models according to different hydrodynamic problems. Different numerical methods are applied to solve the related differential equations.
Continuity Equation

$$
\frac{\partial \rho}{\partial t}+\nabla \cdot(\rho \mathbf{U})=0
$$

where $\rho$ is the density, $\nabla$ is the Hamilton operator, and $\mathbf{U}$ is the velocity vector.

Momentum Equation

$$
\frac{d}{d t}(\rho \mathbf{U})=-\nabla P+\nabla \cdot(\boldsymbol{\tau})+\mathbf{F}
$$


where $P$ is the pressure, $\boldsymbol{\tau}$ is the viscous-stress tensor, and $\mathbf{F}$ is the additional volume stress.

\section{Energy Equation}

$$
\frac{d}{d t}(\rho u)+P(\nabla \cdot \mathbf{U})=\nabla \cdot\left(k_{d} \nabla T\right)+\Phi
$$

where $u$ is the internal energy, $k_{d}$ is the thermal conductivity, $T$ is the temperature, and $\Phi$ is the viscous dissipation term.

3.2. The Turbulent Model. The turbulent $k-\varepsilon$ model is a semiempirical formula summed up from the experiment. It determines the viscosity coefficient by solving the $k$ equation and the $\varepsilon$ equation; then the turbulence stresses are determined.

The functions of standard $k-\varepsilon$ model [19] are calculated as

$$
\begin{aligned}
\frac{\partial(\rho k)}{\partial t}+\frac{\partial\left(\rho k u_{i}\right)}{\partial x_{i}}= & \frac{\partial}{\partial x_{j}}\left[\left(\mu+\frac{\mu_{t}}{\sigma_{k}}\right) \frac{\partial k}{\partial x_{j}}\right]+G_{k}+G_{b} \\
& -\rho \varepsilon-Y_{M}+S_{k} \\
\frac{\partial(\rho \varepsilon)}{\partial t}+\frac{\partial\left(\rho \varepsilon u_{i}\right)}{\partial x_{i}}= & \frac{\partial}{\partial x_{j}}\left[\left(\mu+\frac{\mu_{t}}{\sigma_{\varepsilon}}\right) \frac{\partial \varepsilon}{\partial x_{j}}\right] \\
& +C_{1 \varepsilon} \frac{\varepsilon}{k}\left(G_{k}+C_{3 \varepsilon} G_{b}\right) \\
& -C_{2 \varepsilon} \rho \frac{\varepsilon^{2}}{k}+S_{\varepsilon}
\end{aligned}
$$

where $k$ is turbulent kinetic energy, $\varepsilon$ is turbulent dissipation rate. $C_{1 \varepsilon}, C_{2 \varepsilon}$, and $C_{3 \varepsilon}$ are constants: $C_{1 \varepsilon}=1.44, C_{2 \varepsilon}=1.92$, and $C_{3 \varepsilon}=0.09 . \sigma_{k}$ and $\sigma_{\varepsilon}$ possess the significance of turbulent Prandtl numbers of $k$ and $\varepsilon$ for the transport processes: $\sigma_{k}=1.0$ and $\sigma_{\varepsilon}=1.3 . u_{i}$ is the mean velocity component. $S_{k}$ and $S_{\varepsilon}$ are user-defined source items. $\mu_{t}$ is the turbulent eddy viscosity: $\mu_{t}=\rho C_{\mu} k^{2} / \varepsilon$, where $C_{\mu}$ is suggested as a constant having the value 0.09 . $\mu$ is the dynamic viscosity. $G_{b}$ is the turbulent kinetic energy due to buoyancy. $G_{k}$ is the stress source term due to velocity gradient. $Y_{M}$ is the pulsation expansion term in compressible turbulent flow.

The functions of realizable $k-\varepsilon$ model [21] are defined as

$$
\begin{aligned}
\frac{\partial(\rho k)}{\partial t}+\frac{\partial\left(\rho k u_{j}\right)}{\partial x_{i}}= & \frac{\partial}{\partial x_{j}}\left[\left(u+\frac{u_{t}}{\sigma_{k}}\right) \frac{\partial k}{\partial x_{j}}\right]+G_{k} \\
& +G_{b}-\rho \varepsilon-Y_{M}+S_{k} \\
\frac{\partial(\rho \varepsilon)}{\partial t}+\frac{\partial\left(\rho \varepsilon u_{i}\right)}{\partial x_{i}}= & \frac{\partial}{\partial x_{j}}\left[\left(\mu+\frac{\mu_{t}}{\sigma_{\varepsilon}}\right) \frac{\partial \varepsilon}{\partial x_{j}}\right]+\rho C_{1} E \varepsilon \\
& -\rho C_{2} \frac{\varepsilon^{2}}{k+\sqrt{\nu \varepsilon}}+C_{1 \varepsilon} \frac{\varepsilon}{k} C_{3 \varepsilon} G_{b} \\
& +S_{\varepsilon},
\end{aligned}
$$

where $E=\sqrt{S_{i j} S_{i j}}, S_{i j}$ is the velocity strain rate tensor expressed as $S_{i j}=1 / 2\left(\partial u_{i} / \partial x_{j}+\partial u_{j} / \partial x_{i}\right)$, $v$ is the kinematic viscosity, and $C_{1}$ and $C_{2}$ are constants in numerical simulation.

The $k$ equation in the realizable $k$ - $\varepsilon$ turbulence model is similar to the standard $k$ equation. The constant of the turbulence viscosity formula is changed in the former to satisfy the realistic conditions. Besides, the source term in the $\varepsilon$ equation is no longer related to $G_{k}$. The Reynolds stress term does not appear in the equation, which is good for representing the transfer of the energy spectrum between different scales.

3.3. Boundary Conditions. The boundary conditions of simulation have been shown as follows:

(1) The solver choice is a three-dimensional form based on pressure, implicit difference scheme, and activation energy equation.

(2) The realizable $k-\varepsilon$ turbulence model is selected to carry out the numerical calculations, which is suitable for helically coiled tube.

(3) The near wall adopts the Standard-Wall-Functions treatment.

(4) The SIMPLE algorithm is adopted for the coupling of pressure and velocity.

(5) Relaxation factor of pressure is set as 0.3. Residual energy is set as $10^{-6}$.

(6) Momentum, energy, and turbulent flow are applied as second-order upwind scheme.

The entry condition is applied as velocity inlet. According to the actual working conditions, the reference inlet velocity is $12.45 \mathrm{~m} / \mathrm{s}$, which is calculated by the maximum flux of deicing fluid. Then, four different inlet velocities are set into simulation. The heating boundary conditions of the tube wall are adopted for uniform wall temperature, which is constant, $373 \mathrm{~K}$. No wall-slip condition is adapted to wall surface. The outlet is set as pressure outlet. The temperature of work medium at entrance is set as $278 \mathrm{~K}$.

To investigate the influence of high viscosity deicing fluid on heat transfer in TCHC, water is simulated firstly for contrast. As complex ethylene-glycol-based mixture, deicing fluid is simplified by its main component, ethylene glycol. The model of UDF is used to compile the viscosity-temperature function of ethylene glycol. The other physical parameters needed are set as constants, like density $\rho$, specific heat capacity $c_{P}$, and thermal conductivity $k_{d}$. The material of tube is steel 310. The physical parameters are shown in Table 3.

\section{Simulation with Water}

On the basis of actual working conditions, the inlet velocities are set as $5 \mathrm{~m} / \mathrm{s}, 7.5 \mathrm{~m} / \mathrm{s}, 10 \mathrm{~m} / \mathrm{s}, 12.5 \mathrm{~m} / \mathrm{s}$, and $15 \mathrm{~m} / \mathrm{s}$ to calculate the outlet temperature of TCHC and the original tube. As ideal fluid, water is chosen as contrast to deicing fluid on the influence of viscosity. The outlet temperature is selected as the evaluation index. When outlet temperature is higher, the heat transfer performance is better. 
TABLE 3: The physical parameters of work medium and material of tube.

\begin{tabular}{lccc}
\hline Work medium & Water & Ethylene glycol & Steel 310 \\
\hline $\begin{array}{l}\text { Density } \\
\rho\left(\mathrm{kg} \cdot \mathrm{m}^{-3}\right)\end{array}$ & 998.2 & 1111.4 & 8030.0 \\
\hline $\begin{array}{l}\text { Thermal conductivity } \\
k_{d}\left(\mathrm{~W} \cdot \mathrm{m}^{-1} \cdot \mathrm{K}^{-1}\right)\end{array}$ & 0.600 & 0.252 & 16.270 \\
\hline $\begin{array}{l}\text { Specific heat capacity } \\
c_{p}\left(\mathrm{~J} \cdot \mathrm{kg} \cdot \mathrm{K}^{-1}\right)\end{array}$ & 4182.00 & 2415.00 & 502.48 \\
$\begin{array}{l}\text { Dynamic viscosity } \\
\mu\left(\mathrm{kg} \cdot \mathrm{m}^{-1} \cdot \mathrm{s}^{-1}\right)\end{array}$ & 0.001003 & & \\
\hline
\end{tabular}

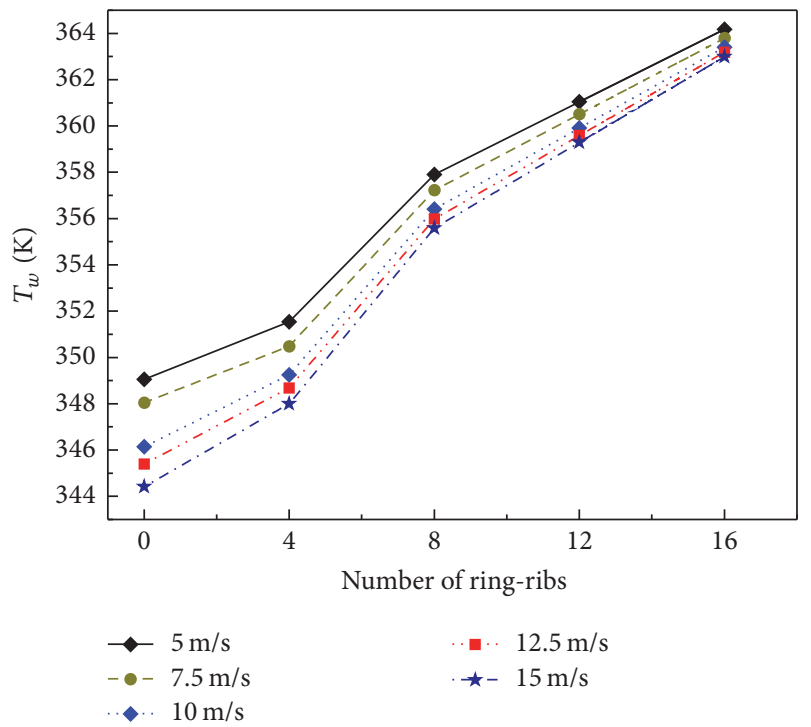

Figure 5: The outlet temperature of water in TCHC with different numbers of ring-ribs.

4.1. Number of Ring-Ribs. Considering how the number of ring-ribs affects heat transfer, the outlet temperature of water $\left(T_{w}\right)$ is analyzed in TCHC and the original tube under different inlet velocities. The results of heat transfer process are summarized in Figure 5.

As seen in Figure 5, all the outlet temperatures of NO. 2, NO. 3, and NO. 4 tubes are higher than that of NO. 1 tube. When the number of ring-ribs increases, the temperature rise tends to flatten out. Consequently, outlet temperature cannot keep rising when the number of ring-ribs exceeds a certain limit. Ring-rib changes the flow state by increasing the radial velocity. The temperature field basically remains radial. Based on field synergy principle, ring-rib can enhance heat transfer by improving the coordination between the velocity and temperature fields.

4.2. Inlet Velocity. From the above discussion, the outlet temperature is affected by inlet velocity. To investigate the influence of different velocities, the outlet temperature differences of water $\left(\Delta T_{w}\right)$ are displayed in Figure 6.

$$
\Delta T_{w}=T_{w_{i}}-T_{w_{j}},
$$

where $i, j$ are the numbers of different inlet velocities.

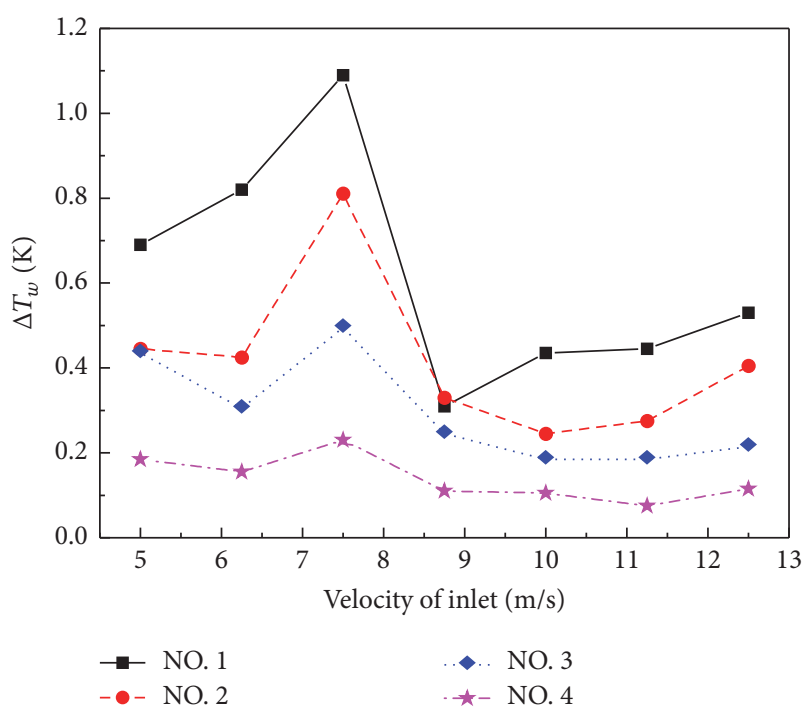

FIGURE 6: The outlet temperature difference trend of water at different inlet velocities.

TABLE 4: The viscosity with the changing temperature of ethylene glycol.

\begin{tabular}{llllllll}
\hline Temperature $(\mathrm{K})$ & 267 & 273 & 289 & 293 & 313 & 333 & 373 \\
\hline Viscosity $(\mathrm{mPa} \cdot \mathrm{s})$ & 86.9 & 53.5 & 25.7 & 22.1 & 10.2 & 5.2 & 1.7 \\
\hline
\end{tabular}

Seen from Figure $6, \Delta T_{w}$ of the NO. 1 tube is higher than those of TCHC, especially at low inlet velocity $(<10 \mathrm{~m} / \mathrm{s})$. To the same tube, when the inlet velocity increases, the outlet temperature gets lower. Water takes less time to pass through the tube. The shortened heating time directly affects the outlet temperature.

$\Delta T_{w}$ of all tubes tends to be flat within a high velocity range in Figure 6. $\Delta T_{w}$ of TCHC changes not as greatly as that of the original tube. Because of ring-rib, TCHC is less affected by the inlet velocity compared with the original tube.

\section{Simulation with Deicing Fluid}

5.1. Viscosity-Temperature Fitting of Deicing Fluid. Deicing fluid is complex ethylene-glycol-based mixture. The thermophysical properties change nonlinearly with temperature. Thus, deicing fluid is simplified by the main component, ethylene glycol. Referring to the experiment, the variation between viscosity and temperature is demonstrated in Table 4.

Considering different function forms, the fitting of power function is most consistent with the actual point. The viscosity of ethylene glycol decreases nonlinearly with the increasing of temperature. The fitting curve is displayed in Figure 7. Equation (7) is derived from the relationship between viscosity and temperature.

The power function of fitting is calculated as

$$
\mu=8 \times 10^{29} \cdot T^{-11.56} .
$$




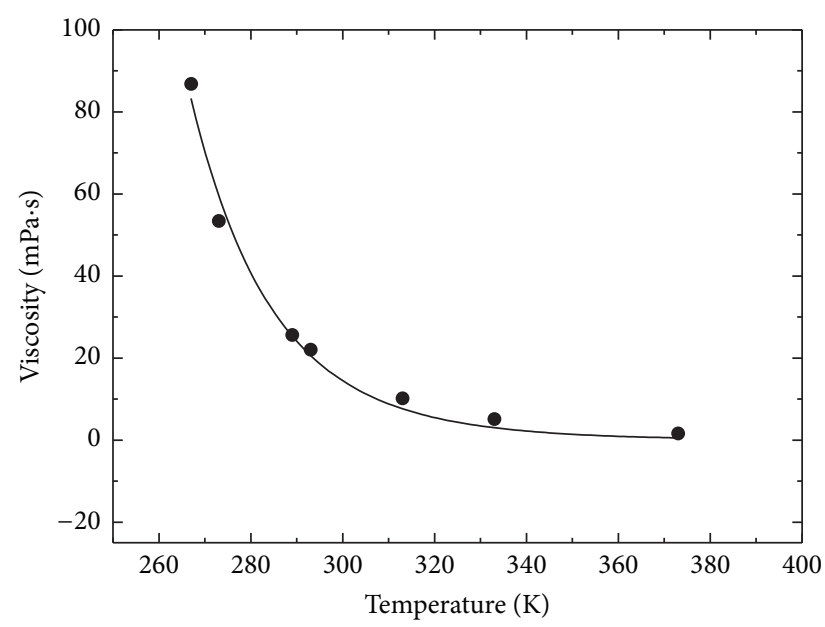

FIgURE 7: The fitting of ethylene glycol's viscosity-temperature.

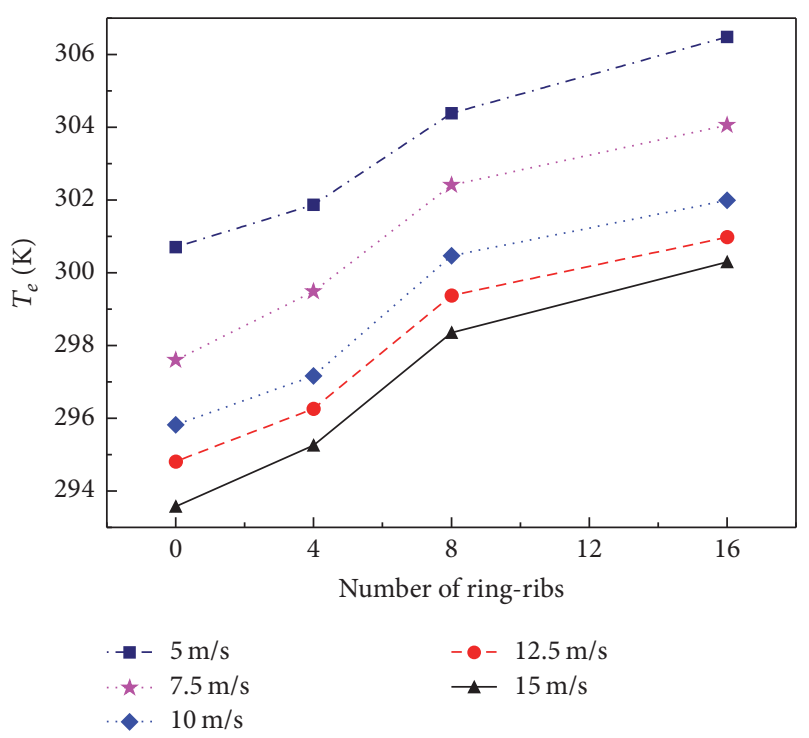

FIGURE 8: The outlet temperature of ethylene glycol in TCHC with different numbers of ring-ribs.

5.2. Number of Ring-Ribs. The outlet temperature of ethylene glycol $\left(T_{e}\right)$ is presented in Figure 8. $T_{e}$ gradually rises when the number of ring-ribs increases. Compared with Figure 5, $T_{e}$ rises less than $T_{w}$ in general. High viscosity is inversely proportional to heat transfer because it thickens the boundary layer of fluid. When the inlet velocity increases, $T_{e}$ generally decreases because the heating time is shortened. Moreover, for both ethylene glycol and water, TCHC can achieve the heat transfer enhancement.

5.3. Inlet Velocity. The temperature difference of ethylene glycol $\left(\Delta T_{e}\right)$ changes with inlet velocity. The tendencies of $\Delta T_{e}$ are shown in Figure 9.

Similarly to water, $\Delta T_{e}$ can be calculated as

$$
\Delta T_{e}=T_{e_{i}}-T_{e_{j}} .
$$

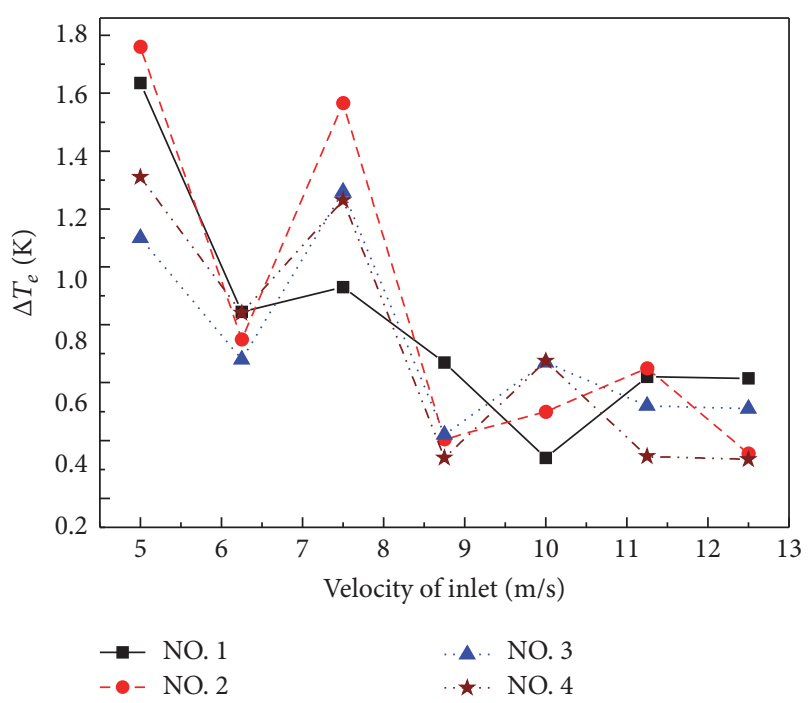

FIGURE 9: The outlet temperature difference trend of ethylene glycol at different inlet velocities.

Seen in Figure 9, because of the power function relation between high viscosity and temperature, $\Delta T_{e}$ varies greatly more than $\Delta T_{w}$ compared with Figure 6 (especially $7.5 \mathrm{~m} / \mathrm{s}-10 \mathrm{~m} / \mathrm{s}) . \Delta T_{e}$ of all tubes tends to be flat within a high velocity range.

\section{Discussions by Field Synergy Principle}

Field synergy principle is a novel theory for heat transfer enhancement. The coordination between velocity field and temperature field has a positive influence on the heat transfer enhancement $[5,6]$. Equation (9) gives a more general insight on convective heat transfer. There are two ways to enhance heat transfer: (a) increasing Reynolds and/or Prandtl number; (b) increasing the value of the dimensionless integration [4].

$$
\mathrm{Nu}_{x}=\operatorname{Re}_{x} \operatorname{Pr} \int_{0}^{1}(\bar{U} \cdot \nabla \bar{T}) d \bar{y} .
$$

$\bar{U} \cdot \nabla \bar{T}$ in the dimensionless integration in (9) can be expressed as

$$
\bar{U} \cdot \nabla \bar{T}=|\bar{U}||\bar{T}| \cos \beta,
$$

where $\beta$ is the included angle between the velocity vector and the temperature gradient (heat flow vector).

Figure 10 sketches the outlet velocity fields of NO. 1 and No. 4 tubes at the same inlet velocity. The movement in the NO. 4 tube is more obvious than that in NO. 1 tube. Because of the ring-rib, the fluid movement is strengthened from wall to center in TCHC. Ring-rib changes velocity field by breaking the thickness of the flow boundary layer. As seen in Figure 10, the flow in TCHC mixes well, so the temperature distribution becomes more uniform. TCHC has efficiently improved the coordination of velocity and temperature fields. For this reason, TCHC can achieve the heat transfer enhancement. 


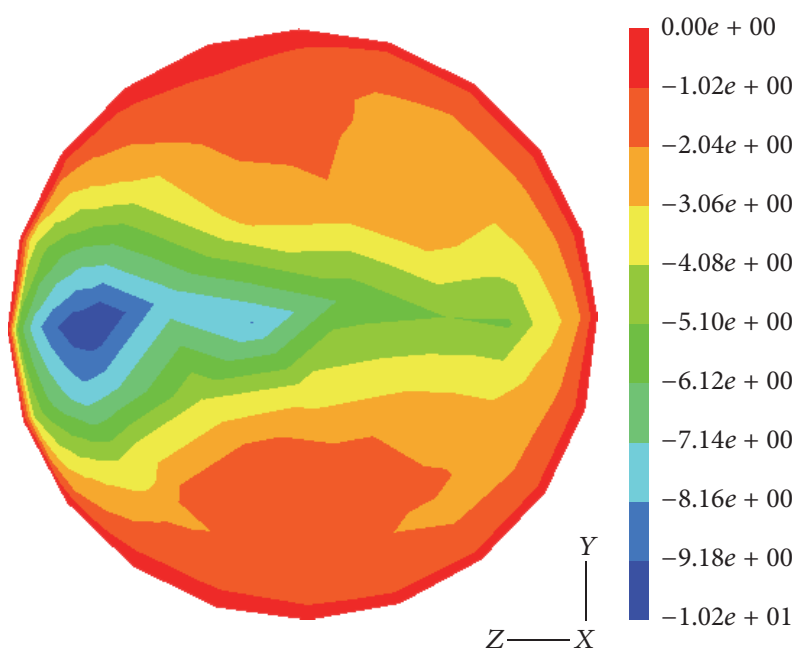

(a)

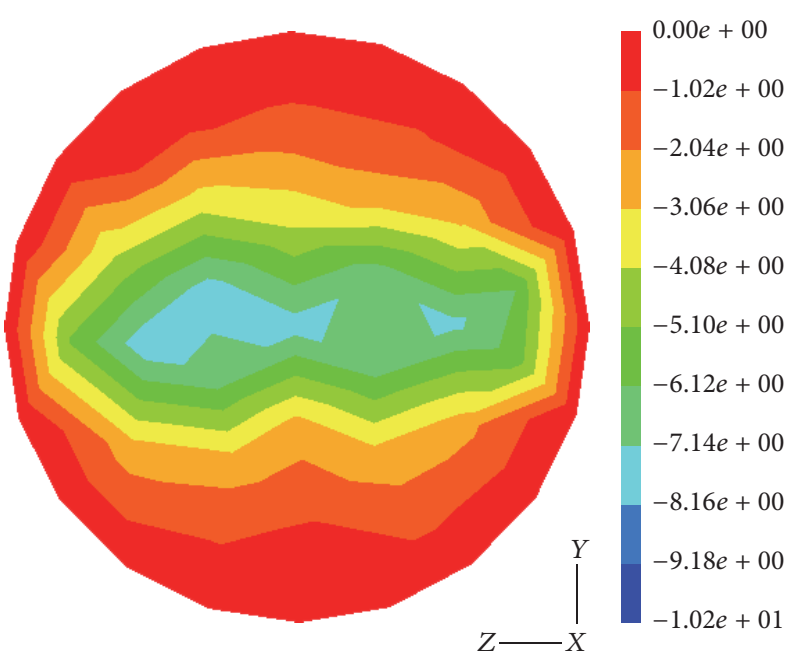

(b)

Figure 10: The outlet velocity field. (a) NO. 1 tube and (b) NO. 4 tube.

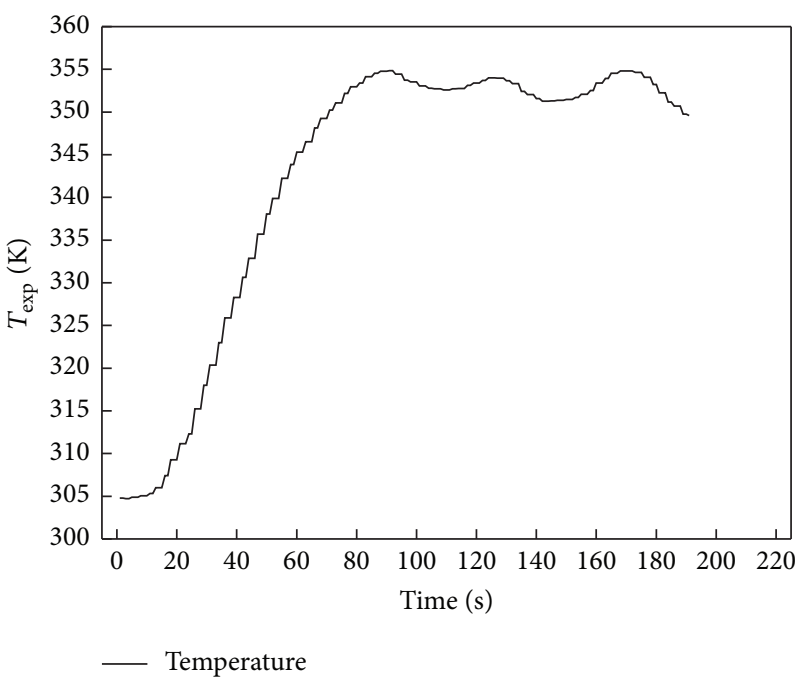

FIGURE 11: Heating experiment of the original tube.

\section{Heating Experiment}

The heating experiment of the original tube is conducted. The experimental results are demonstrated in Figure 11. At steady working condition, the outlet temperature $\left(T_{\exp }\right)$ is basically stable at $350 \mathrm{~K}$.

The error $(\delta)$ between experiment and simulation can be expressed as

$$
\delta=\frac{\left|T_{w}-T_{\exp }\right|}{T_{\exp }} \times 100 \%
$$

Through calculation, $\delta$ is less than $5 \%$; thus the experiment verifies the correctness of numerical methods. At the same working conditions, the outlet temperature of TCHC increases by $3.76 \%$ compared with the experimental data.
Therefore, TCHC has better heat transfer performance than the original tube.

\section{Conclusion}

The original helically coiled tube is reshaped into TCHC based on field synergy principle. The heat transfer of deicing fluid is analyzed in TCHC and the original tube. Water is selected as a contrast to deicing fluid on the influence of viscosity. The outlet temperature is chosen as the evaluation index. The number of ring-ribs and inlet velocity are two key parameters to the heat transfer performance. The results are summarized as follows:

(1) Both number of ring-ribs and inlet velocity can affect heat transfer greatly. For water and ethylene glycol, the outlet temperature rises when the number of ring-ribs increases to a certain limit. However, the increasing of velocity reduces heating time, which results in lower outlet temperature.

(2) High viscosity is inversely proportional to heat transfer because viscosity thickens boundary layer of fluid. The outlet temperature rise of ethylene glycol is not as obvious as that of water.

(3) Both water and ethylene glycol in TCHC are more efficiently heated compared with the original tube. Ring-rib changes the velocity field based on field synergy principle. In consequence, TCHC improves the coordination between velocity and temperature fields.

(4) The error between experiment and simulation is less than $5 \%$. The outlet temperature of TCHC increases by $3.76 \%$ compared to that of the original tube.

In general, TCHC has enhanced heat transfer of high viscosity deicing fluid. Therefore, TCHC is beneficial to deicing efficiency and flight punctuality. 


\section{Nomenclature}

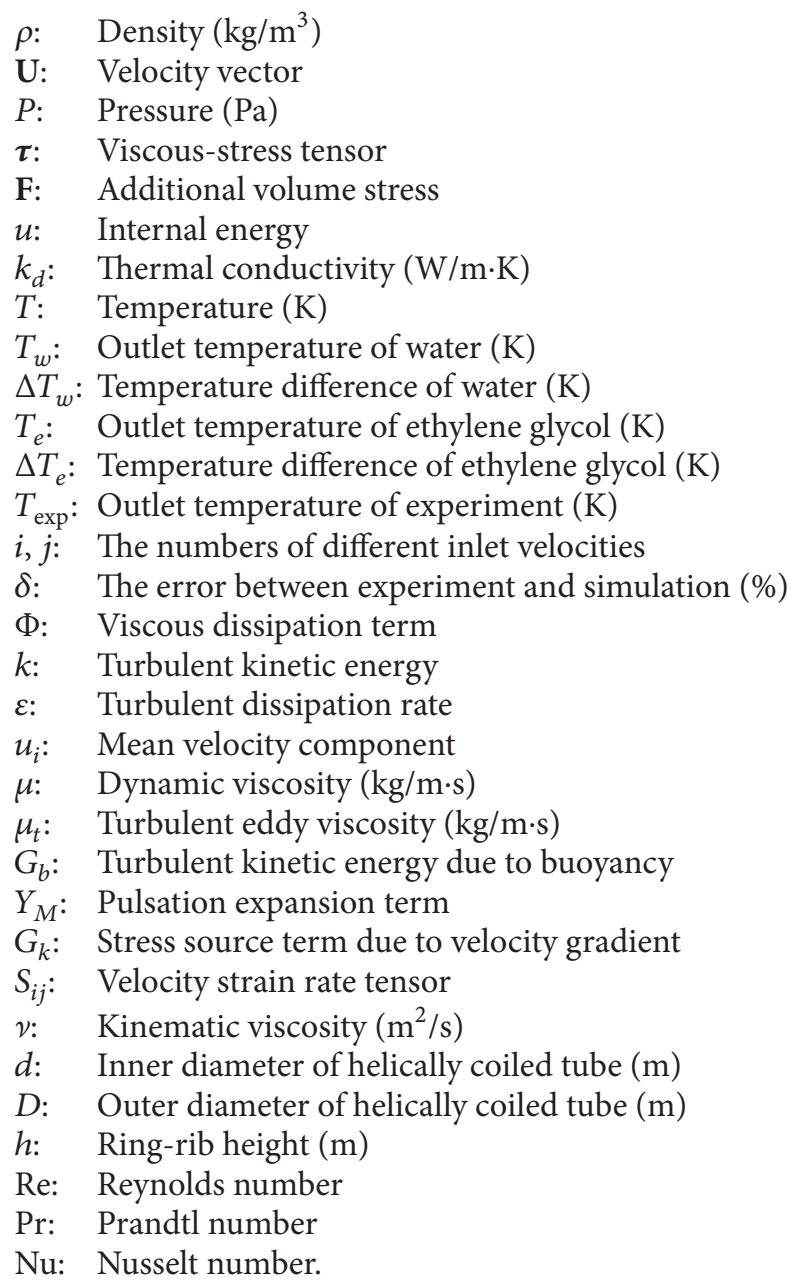

\section{Conflicts of Interest}

The authors declare that they have no conflicts of interest.

\section{Acknowledgments}

This research is financially supported by the Natural Science Foundation of Tianjin (15JCQNJC42900), National Natural Science Foundation of China (51505483), and the Fundamental Research Funds for the Central Universities (3122013C012).

\section{References}

[1] S. Sun and L. W. Wang, "Study on the instant heat structure for aircraft de-icing fluid," Machine Tool \& Hydraulics, vol. 38, no. 8, pp. 59-60, 2010.

[2] AMS 1424J De-icing/Anti-icing fluid Aircraft, SAE Type I, 2006.

[3] AMS 1428E Fluid, Aircraft De-icing/Anti-icing, Non-Newtonian (Pseudo-plastic), SAE Type II, III and IV, 2006.

[4] Z. Y. Guo, W. Q. Tao, and R. K. Shah, "The field synergy (coordination) principle and its applications in enhancing single phase convective heat transfer," International Journal of Heat and Mass Transfer, vol. 48, no. 9, pp. 1797-1807, 2005.
[5] Z. Y. Guo, D. Y. Li, and B. X. Wang, "A novel concept for convective heat transfer enhancement," International Journal of Heat and Mass Transfer, vol. 41, no. 14, pp. 2221-2225, 1998.

[6] Z. Guo, "Mechanism and control of convective heat transfercoordination of velocity and heat flow fields," Chinese Science Bulletin, vol. 46, no. 7, pp. 596-599, 2001.

[7] S. Wang, Z. Y. Guo, and Z. X. Li, "Heat transfer enhancement by using metallic filament insert in channel flow," International Journal of Heat and Mass Transfer, vol. 44, no. 7, pp. 1373-1378, 2001.

[8] W. Liu, Z. Liu, and Z. Guo, "Physical quantity synergy in laminar flow field of convective heat transfer and analysis of heat transfer enhancement," Chinese Science Bulletin, vol. 54, no. 19, pp. 35793586, 2009.

[9] W. Liu, Z. C. Liu, and S. Y. Huang, "Physical quantity synergy in the field of turbulent heat transfer and its analysis for heat transfer enhancement," Chinese Science Bulletin, vol. 55, no. 23, pp. 2589-2597, 2010.

[10] W. Liu, Z. C. Liu, and L. Ma, "Application of a multi-field synergy principle in the performance evaluation of convective heat transfer enhancement in a tube," Chinese Science Bulletin, vol. 57, no. 13, pp. 1600-1607, 2012.

[11] W. R. Dean, "XVI. Note on the motion of fluid in a curved pipe," The London, Edinburgh, and Dublin Philosophical Magazine and Journal of Science, vol. 4, no. 20, pp. 208-223, 1927.

[12] W. Dean, "LXXII. he stream-line motion of fluid in a curved pipe (second paper)," The London, Edinburgh, and Dublin Philosophical Magazine and Journal of Science, vol. 5, no. 30, pp. 673-695, 1928.

[13] E. C. Romão, "Efficient alternative for construction of the linear system stemming from numerical solution of heat transfer problems via FEM," Mathematical Problems in Engineering, vol. 2016, Article ID 1614324, pp. 1-7, 2016.

[14] S. M. Mehdi, M. Akhtar, A. Hussain, D. S. Alothmany, and S. Aziz, "CFD study of liquid sodium inside a wavy tube for laminar convectors: effect of reynolds number, wave pitch, and wave amplitude," Mathematical Problems in Engineering, vol. 2016, Article ID 6146195, p. 17, 2016.

[15] R. Palanichamy and P. Nagaraj, "Numerical simulation of laminar heat transfer in aluminium circular tube with internal longitudinal fins," International Journal of Modelling and Simulation, vol. 30, no. 2, pp. 204-210, 2015.

[16] E. Tian, Y.-L. He, and W.-Q. Tao, "Numerical simulation of finned tube bank across a staggered circular-pin-finned tube bundle," Numerical Heat Transfer, Part A: Applications, vol. 68, no. 7, pp. 737-760, 2015.

[17] C. X. Lin and M. A. Ebadian, "Developing turbulent convective heat transfer in helical pipes," International Journal of Heat and Mass Transfer, vol. 40, no. 16, pp. 3861-3873, 1997.

[18] I. Di Piazza and M. Ciofalo, "Numerical prediction of turbulent flow and heat transfer in helically coiled pipes," International Journal of Thermal Sciences, vol. 49, no. 4, pp. 653-663, 2010.

[19] W. P. Jones and B. E. Launder, "The prediction of laminarization with a two-equation model of turbulence," International Journal of Heat and Mass Transfer, vol. 15, no. 2, pp. 301-314, 1972.

[20] S. B. Pope, Turbulent Flows, Cambridge University Press, Cambridge, UK, 2000.

[21] T.-H. Shih, W. W. Liou, A. Shabbir, Z. Yang, and J. Zhu, "A new $k-\epsilon$ eddy viscosity model for high reynolds number turbulent flows," Computers \& Fluids, vol. 24, no. 3, pp. 227-238, 1995. 
[22] M. Lateb, C. Masson, T. Stathopoulos, and C. Bédard, "Comparison of various types of $k-\varepsilon$ models for pollutant emissions around a two-building configuration," Journal of Wind Engineering \& Industrial Aerodynamics, vol. 115, pp. 9-21, 2013.

[23] T. J. Hüttl and R. Friedrich, "Influence of curvature and torsion on turbulent flow in helically coiled pipes," International Journal of Heat and Fluid Flow, vol. 21, no. 3, pp. 345-353, 2000.

[24] A. Zachár, "Investigation of natural convection induced outer side heat transfer rate of coiled-tube heat exchangers," International Journal of Heat and Mass Transfer, vol. 55, no. 25-26, pp. 7892-7901, 2012.

[25] F. M. White, Fluid Mechanics, McGraw-Hill, New York, NY, USA, 1994. 


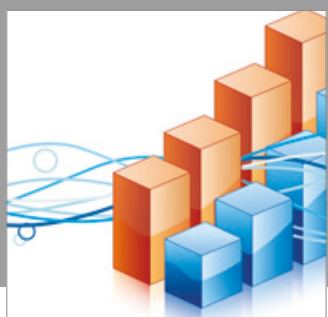

Advances in

Operations Research

vatersals

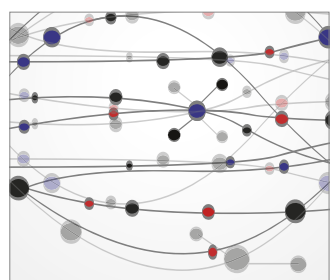

\section{The Scientific} World Journal
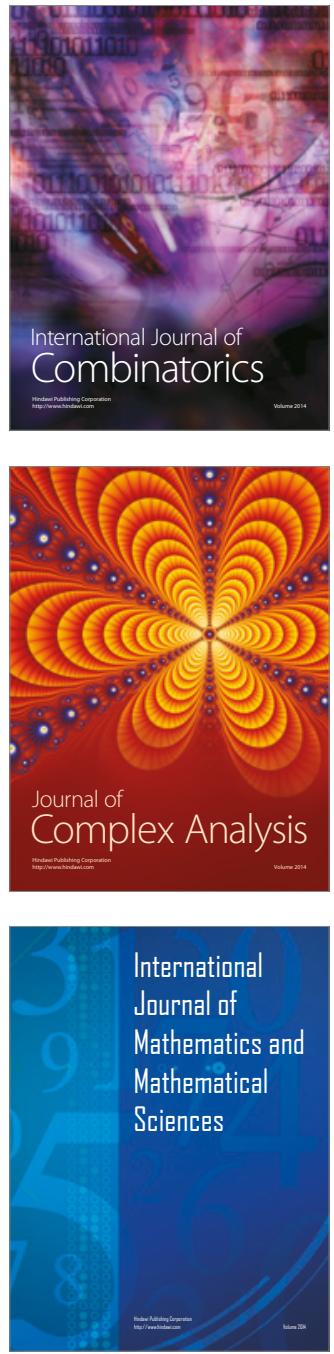
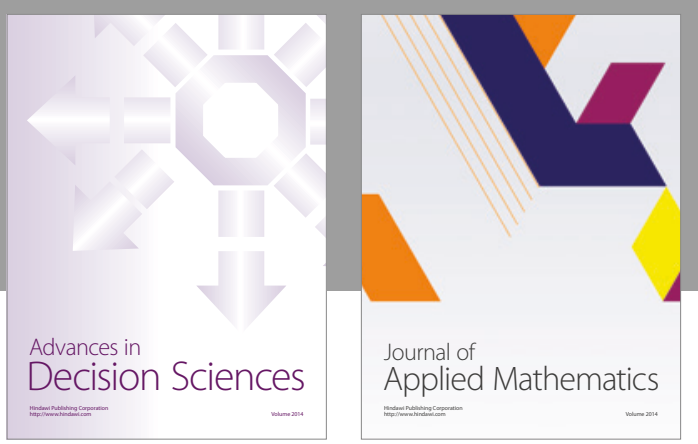

Algebra

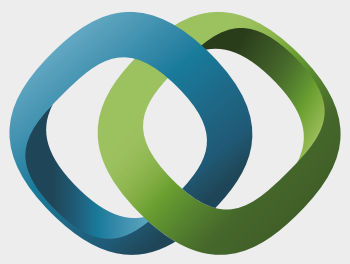

\section{Hindawi}

Submit your manuscripts at

https://www.hindawi.com
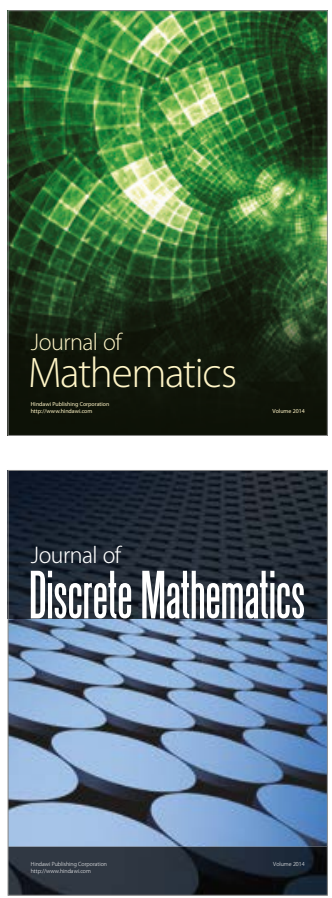

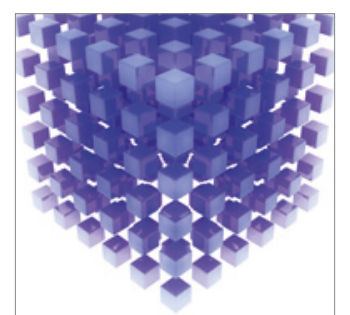

Mathematical Problems in Engineering
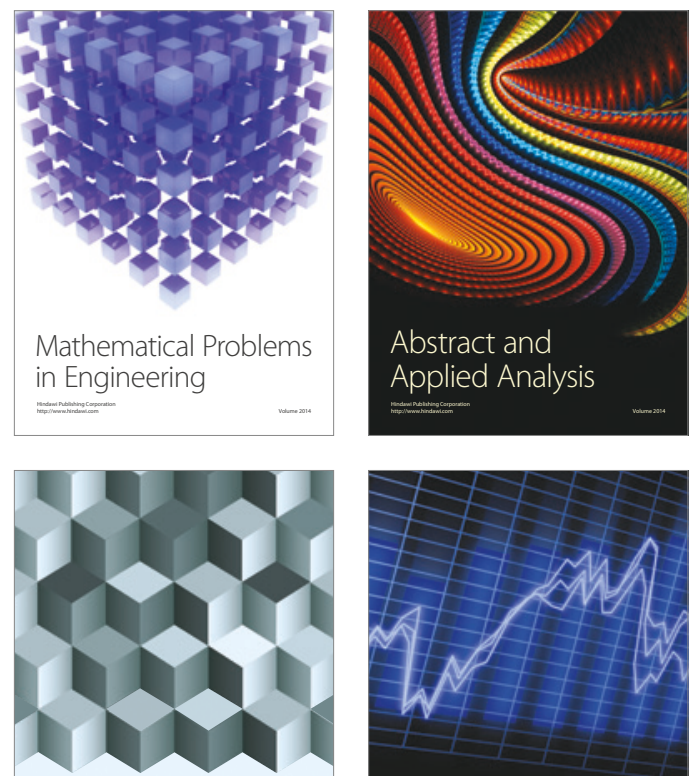

Journal of

Function Spaces

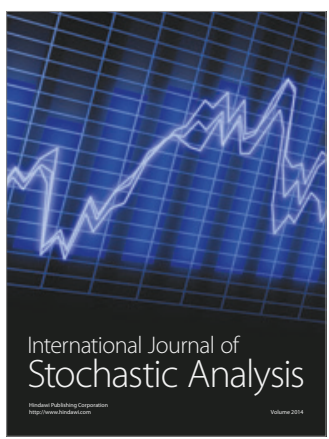

Probability and Statistics
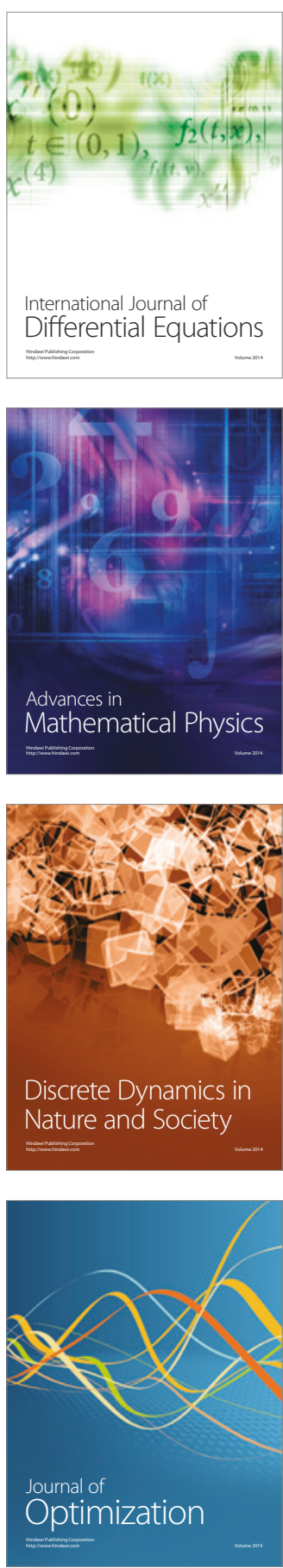